\title{
ALTERAÇÕES BIOQUÍMICAS E ACÚMULO EM PACUS (Metynnis argenteus) EXPOSTOS AO PACLOBUTRAZOL
}

\author{
Claudio Martín Jonsson*; Vera Lúcia Ferracini; Lourival Costa Paraíba; Maurício Rangel; Sandra \\ Regina Aguiar
}

Embrapa Meio Ambiente, C.P. 69 - CEP:13820-000 - Jaguariúna, SP.

*Autor correspondente <jonsson@cnpma.embrapa.br>

\begin{abstract}
RESUMO: O paclobutrazol, um regulador de crescimento vegetal com propriedades fungicidas, é muito utilizado na região Nordeste do Brasil em áreas de produção de frutíferas. Este composto pode permanecer estável por vários meses após sua aplicação no solo, sendo sua meia vida na água superior a três semanas. Corpos de água superficiais nas proximidades das culturas são portanto susceptíveis de serem contaminados pelo paclobutrazol, com o risco de promover efeitos adversos para a fauna aquática e para a saúde humana. No presente trabalho foi estudada a bioconcentração e algumas alterações bioquímicas, medidas pela atividade das enzimas transaminase glutâmico-oxalacética (TGO), lactato desidrogenase (LDH) e glutationa Stransferase (GST) em tecidos do peixe pacú prata (Metynnis argenteus), exposto ao paclobutrazol durante 28 dias. O paclobutrazol aumentou a atividade de LDH e GST no fígado para as concentrações de $3 \mathrm{mg} \mathrm{L}^{-1}$ ( $28^{\circ}$ dia de exposição) e $30 \mathrm{mg} \mathrm{L}^{-1}$ ( $7^{\circ}$ dia de exposição), respectivamente. No $28^{\circ}$ dia, a concentração de paclobutrazol no músculo atingiu níveis residuais de $166 \mathrm{mg} \mathrm{kg}^{-1}$ para a concentração média determinada na água de 2,7 $\mathrm{mg} \mathrm{L}^{-1}$, estimando-se um fator de bioconcentração (FBC) para a situação de equilíbrio equivalente a 73. A medida da atividade de LDH e GST pode ser utilizada como biomarcador de exposição ao paclobutrazol. Esta exposição deve estar em situações extremas para proporcionar risco significativo em humanos que se alimentam de peixes contendo resíduos do agroquímico.
\end{abstract}

Palavras-chave: bioconcentração, peixe, toxicidade

\section{BIOCHEMICAL CHANGES AND ACCUMULATION IN "PACU” FISH (Metynnis argenteus) EXPOSED TO PACLOBUTRAZOL}

\begin{abstract}
Paclobutrazol is a plant growth regulator with fungicide properties which is widely used in Brazilian Northeast orchards. This chemical remains stable in the soil for several months and its half-life in water is longer than three weeks. Because of these characteristics, the aquatic contamination by paclobutrazol may occur, potentially harming aquatic fauna and human health. This work evaluates the bioconcentration and the activities of the enzymes: glutamic oxaloacetic transaminase (GOT), lactate dehydrogenase (LDH) and glutathione S-transferase (GST) in the Brazilian fish "pacu prata" (Metynnis argenteus) exposed to paclobutrazol for 28 days. Results showed a significant increase in the enzymatic activity of LDH and GST in liver, for concentrations equals to $3 \mathrm{mg} \mathrm{L}^{-1}\left(28^{\circ}\right.$ day exposure) and $30 \mathrm{mg} \mathrm{L}^{-1}\left(7^{\circ}\right.$ day exposure), respectively. Paclobutrazol residues in muscle reached $166 \mathrm{mg} \mathrm{kg}^{-1}$ at the end of the period of exposure for the measured concentration in the water equivalent to $2.7 \mathrm{mg} \mathrm{L}^{-1}$. The bioconcentration factor estimated for the steady state condition was 73. It was concluded that the measure of LDH and GST activities could be used as biomarkers of paclobutrazol exposure. This exposure may already be in extreme conditions to cause significant risk to humans consuming contaminated fish.
\end{abstract}

Key words: bioconcentration, fish, toxicity

\section{INTRODUÇÃO}

A complexidade de ecossistemas aquáticos dificulta a avaliação e predição de efeitos de agroquímicos no meio ambiente. Isto tem trazido complicações na determinação de concentrações máximas permissíveis desses agentes em corpos de água receptores.

A necessidade de entender e predizer os efeitos dos agroquímicos tem promovido a pesquisa de vários indicadores fisiológicos e bioquímicos de compostos tóxicos indutores de estresse (Jimenez \& Stegeman, 1990; Mehrle \& Mayer, 1985). O conceito básico que sustenta a utilização de bioindicadores de poluição ambiental por agroquímicos baseia-se no fato que os distúrbios no meio ambiente levam inicialmente à perturbação de uma reação bioquímica em um determinado organismo. A observação destas alterações bioquímicas com antecedência, possibilita a identificação de problemas ambientais antes que o ecossistema aquático como um todo seja afetado (Bucheli \& Fent, 1995).

A maior parte dos agroquímicos usados no controle de pragas e como reguladores do crescimento vegetal são compostos que possuem baixa solubilidade em água e alta afinidade por gorduras sendo, portanto, facilmente absorvidos pelo organismo animal. Quando a velocidade de absorção do 
composto através da água excede a velocidade de eliminação do mesmo, ocorre o acúmulo, processo conhecido como bioconcentração (Spacie \& Hamelink, 1985). Tal fenômeno é considerado de grande importância tanto na manifestação dos efeitos subletais de agroquímicos em organismos não alvo, quanto na prevenção de contaminação de fontes protéicas de consumo humano (Kanazawa, 1981).

Denomina-se de fator de bioconcentração (FBC) a relação entre a concentração do composto no tecido do organismo e na água na situação de equilíbrio, podendo este parâmetro também ser calculado por constantes cinéticas. O paclobutrazol, um agroquímico regulador de crescimento vegetal com propriedades fungicidas, é muito utilizado na região Nordeste do Brasil em áreas de produção de frutas para exportação. Estudos têm demonstrado que este composto permanece ativo no solo por muito tempo e que sua meia-vida varia com o tipo de solo e condições climáticas. A meia-vida do paclobutrazol na água é de 24 dias, enquanto que no solo não foi detectada dissipação até a amostragem aos 168 dias após a aplicação do produto (Chand \& Lembi, 1994). Corpos de água superficiais nas proximidades das culturas frutíferas são, portanto, suscetíveis de serem contaminados pelo paclobutrazol com o risco de promover efeitos adversos em organismos que habitam esses compartimentos ambientais.

Este trabalho teve como principal objetivo avaliar a atividade de algumas enzimas em uma espécie de peixe autóctone pertencente à família Characidae, tentando determinar a possibilidade de seu uso como biomarcadoras de contaminação de corpos de água pelo composto. Foi também estudada a bioconcentração do paclobutrazol nesse organismo. As informações sobre o metabolismo do paclobutrazol em peixes foram obtidas com vistas a ampliar a base de dados associada à ação de agroquímicos usados no Brasil sobre organismos nãoalvo.

\section{MATERIAL E MÉTODOS}

\section{Material-teste}

Paclobutrazol ((2RS,3RS)-1-(4-chlorofenil)-4, 4dimetil-2-(1H-1,2,4,triazol-1-yl) pentan-3-ol), sob a forma de suspensão concentrada $\left(\right.$ CULTAR $\left.^{\circledR}\right)$, contendo $250 \mathrm{~g} \mathrm{~L}^{-1}$ de ingrediente ativo.

\section{Organismo-teste}

Peixes pacú prata pesando em média $5 \mathrm{~g}$, foram adquiridos de um fornecedor local e mantidos em água de poço artesiano com temperatura $26-29^{\circ} \mathrm{C} ; \mathrm{pH}$ 7,8; dureza total $36 \mathrm{mg} \mathrm{L}^{-1} \mathrm{CaCO}_{3}$; condutividade 190 $\mu S \mathrm{~cm}^{-1}$. Os peixes foram aclimatados por um período de 10 dias após sua aquisição, em caixas de cimentoamianto com capacidade de $100 \mathrm{~L}$, submetidos a um fotoperíodo de $16 \mathrm{~h}$ luz (500 lux)/8h escuro.

\section{Sistema de exposição}

Os ensaios foram realizados em aquários de vidro com volume de $15 \mathrm{~L}$. A relação biomassa/volume de água foi de aproximadamente $1 \mathrm{~g} \mathrm{~L}^{-1}$. As soluções em concentrações de 3 e $30 \mathrm{mg} \mathrm{L}^{-1}$ de ingrediente ativo foram preparadas a partir de uma suspensão estoque do produto formulado e adicionada à mesma água utilizada durante o período de aclimatação. As exposições foram realizadas em sistema semi-estático com troca das soluções uma vez por semana.

Paralelamente foram preparados aquários contendo somente água isenta do composto que serviram como controle. Os peixes foram submetidos às mesmas condições ambientais descritas anteriormente.

\section{Estudo de alterações bioquímicas}

Seis peixes provenientes de aquários contendo 3 e $30 \mathrm{mg} \mathrm{L}^{-1}$ de paclobutrazol foram retirados no $7^{\circ}, 14^{\circ}$ e $28^{\circ}$ dia de exposição. Os organismos foram sacrificados por decapitação e foi efetuada a separação de parte do tecido muscular e da porção de vísceras abdominais contendo o fígado.

Foi preparado um homogeneizado de tecido na proporção de $0,5 \mathrm{~g}$ tecido $10 \mathrm{~mL}^{-1}$ solução de sacarose $0,25 \mathrm{~mol} \mathrm{~L}^{-1}$. O homogeneizado foi centrifugado em uma centrífuga Sorvall Super T21 durante 20 minutos a $10.000 \times \mathrm{g}$ a uma temperatura de $4^{\circ} \mathrm{C}$. O sobrenadante foi separado para a análise de atividade enzimática e de proteínas. As proteínas foram analisadas através do método de Lowry modificado por Peterson (1977).

A atividade da transaminase glutâmicooxalacética (TGO) foi determinada através da metodologia descrita por Frankel \& Reitman (1963). A mistura de incubação continha $1 \mathrm{~mL}$ de substrato TGO (200 $\mathrm{mmol} \mathrm{L}^{-1}$ de d-L-aspartato; $2 \mathrm{mmol} \mathrm{L}^{-1}$ de acido alfacetoglutárico, em tampão fosfato) e a fonte de enzimas. Após a incubação a $37^{\circ} \mathrm{C}$ por 60 minutos, a reação foi interrompida pela adição de $1 \mathrm{~mL}$ de 2, 4dinitrofenilhidrazina $1 \mathrm{mmol} \mathrm{L^{-1 }}$. A coloração foi desenvolvida pela adição de $10 \mathrm{~mL}$ de $\mathrm{NaOH} 0,4 \mathrm{~mol} \mathrm{~L}^{-1}$ e a leitura da absorvância do piruvato produzido foi realizada a $505 \mathrm{~nm}$.

A atividade da lactato desidrogenase (LDH) foi determinada através do método cinético descrito por Vassault (1983). A mistura de reação consistiu em 2,5 $\mathrm{mL}$ de NADH 0,24 mmol L-1 em Tris/NaCl pH 7,2; $0,5 \mathrm{~mL}$ de piruvato $9,8 \mathrm{mmol}^{-1}$ e uma alíquota do sobrenadante. A diminuição linear da absorvância a 339 $\mathrm{nm}$ foi monitorada durante 2 minutos a temperatura de $25^{\circ} \mathrm{C}$. A atividade foi expressa em $\mu$ moles de $\mathrm{NADH}$ (consumido) minuto-1 $\mathrm{mg}^{-1}$ de proteína, usando-se um coeficiente de extinção de $6,3 \mathrm{mmol} \mathrm{L}^{-1} \mathrm{~cm}^{-1}$.

A atividade da glutationa S-transferase (GST) foi determinada de acordo com o método cinético descrito por Habig et al. (1974). A mistura de reação $(3 \mathrm{~mL})$ contendo $2,8 \mathrm{~mL}$ de $\mathrm{GSH}$ (glutationa reduzida) $1 \mathrm{mmol}$ $\mathrm{L}^{-1}$ em tampão fosfato $\mathrm{pH} 6.5 ; 50 \mu \mathrm{L}$ de 1-cloro-2, 4- 
dinitrobenzeno (CDNB) 0,06 mol L-1 em etanol e uma alíquota adequada de sobrenadante foi incubada durante 3 minutos a $25^{\circ} \mathrm{C}$. O aumento linear da absorvância a $340 \mathrm{~nm}$ foi monitorado e a atividade foi expressa em nmoles de conjugado GSH-CDNB produzido minuto ${ }^{-1}$ $\mathrm{mg}^{-1}$ de proteína usando-se o coeficiente de extinção de $9,6 \mathrm{mmolar}^{-1} \mathrm{~cm}^{-1}$. As leituras das análises foram realizadas em um espectrofotômetro ultravioleta-visível Perkin Elmer Lambda-20.

\section{Estudo da bioconcentração}

Três gramas de tecido muscular provenientes dos peixes amostrados no $7^{\circ}, 14^{\circ}, 21^{\circ}$ e $28^{\circ}$ dias de exposição, foram extraídas em um extrator Omni Solv com $100 \mathrm{~mL}$ de metanol $80 \%$. O metanol foi evaporado a $35^{\circ} \mathrm{C}$ em um rotaevaporador e o $\mathrm{pH}$ foi corrigido para $11 \mathrm{com} \mathrm{NaOH} \mathrm{6,5} \mathrm{mol} \mathrm{L-1.} \mathrm{Foi} \mathrm{realizada} \mathrm{uma} \mathrm{extração}$ líquido-líquido com diclorometano ( $3 \times 50 \mathrm{~mL}$ ). $\mathrm{O}$ diclorometano foi totalmente evaporado e o extrato foi resuspendido em $10 \mathrm{~mL}$ de hexano. Para a quantificação do paclobutrazol foi utilizado um cromatógrafo a gás HP 6890 equipado com coluna capilar HP-5 (30 m x 0,32 mm $x 0,25 \mu \mathrm{m})$ e detector NPD. As condições analíticas foram: programação de temperatura do forno $60^{\circ} \mathrm{C}(1$ $\min ), 60-240^{\circ} \mathrm{C}\left(40^{\circ} \mathrm{C} \mathrm{min}{ }^{-1}\right) ; 240^{\circ} \mathrm{C}(9 \mathrm{~min})$; temperatura do injetor $250^{\circ} \mathrm{C}$; temperatura do detector $250^{\circ} \mathrm{C}$; fluxo de He $2 \mathrm{~mL} \mathrm{~min}^{-1}$.

As amostras de água contendo paclobutrazol foram filtradas em uma membrana Millipore de $0.45 \mu \mathrm{m}$ e a análise foi realizada por cromatografía líquida de alta eficiência (HPLC) em um aparelho Shimadzu modelo LC10A, equipado com detector de arranjo de fotodiodos. As condições de análise foram: coluna C18 (4,6 m x 26 $\mathrm{cm} \times 5 \mu \mathrm{m})$, fase móvel MeOH: $\mathrm{H}_{2} \mathrm{O}(68: 32$, v/v), fluxo de $0,7 \mathrm{~mL} \mathrm{~min}{ }^{-1}$, "loop" de injeção de $50 \mu \mathrm{L}$ e detecção em $227 \mathrm{~nm}$.

A determinação do fator de bioconcentração (FBC), em estado de equilíbrio, foi feita calculando-se o limite da concentração no peixe quando o tempo tende ao infinito, pelas expressões:

$$
F B C=\lim _{t \rightarrow \infty} C_{p}(t)=\frac{k_{1}}{k_{2}}
$$

e

$$
C_{p}(t)=\frac{k_{1}}{k_{2}} C_{a}\left(1-\exp \left(-\left(\frac{k_{2}}{(n+1)}\right) t^{(n+1)}\right)\right)
$$

onde $k_{1}>0$ é a constante de assimilação, $k_{2}>0$ é a constante de eliminação, $n$ é um parâmetro empírico, $C_{a}$ é concentração constante na água e $C_{p}=C_{p}(t)$ é a concentração no peixe ao longo do tempo $t$. A expressão teórica acima para $C_{p}=C_{p}(t)$ generaliza o modelo clássico de bioconcentração. Os parâmetros $k_{1}, k_{2}$ e $n$ foram obtidos por ajuste numérico por mínimos quadrados dos dados experimentais à curva $C_{p}=C_{p}(t)$.

\section{Análise estatística}

O teste $t$ de Student foi utilizado para comparar as diferenças entre os controles e os grupos experimentais. O nível de significância foi estabelecido como sendo $P<0,05$.

\section{RESULTADOS E DISCUSSÃO}

Nas Figuras 1-6 estão apresentados os resultados referentes aos estudos das alterações bioquímicas decorrentes do paclobutrazol em tecido muscular e fígado de pacú prata expostos às concentrações nominais de 3 e $30 \mathrm{mg} \mathrm{L}^{-1}$ durante 28 dias. Os valores nas Figuras 1-6 correspondem às medias de 5 ou 6 análises + desvio padrão.

Após $07^{\circ}$ dia de exposição, ocorreu a mortalidade dos peixes na maior concentração testada, o que não permitiu avaliar as alterações bioquímicas para a concentração de exposição de $30 \mathrm{mg} \mathrm{L}^{-1}$. Não foram observadas alterações significativas nas atividades de TGO provenientes dos dois tecidos durante o período total de exposição (Figuras 1 e 2). A GST do fígado, na exposição à concentração de $30 \mathrm{mg} \mathrm{L}^{-1}$ de paclobutrazol, apresentou um aumento significativo em sua atividade em relação ao controle $(P=0,00067)$. O mesmo fenômeno ocorreu para a LDH do fígado de peixes expostos a $3 \mathrm{mg} \mathrm{L}^{-1}$ de paclobutrazol durante 28 dias, porém o efeito foi bem menos significativo $(P=0,0103)$ que para a GST (Figuras 4 e 6).

A TGO e LDH são enzimas presentes em todos os organismos. Estas enzimas não somente funcionam como enzimas de ligação entre o metabolismo de proteínas e carboidratos, como também servem como indicadoras de condições fisiológicas ou estruturais alteradas. Estas enzimas desempenham um papel importante em condições de estresse ocasionado por agentes químicos, e têm sido objeto de estudos devido à alteração de sua atividade em organismos aquáticos sob exposição aguda ou crônica a agroquímicos (Rao et al., 1990; Reddy et al., 1995; Asztalos et al., 1990), incluindo compostos com ação fungicida (Rojik et al., 1983, Tiedge et al.,1986).

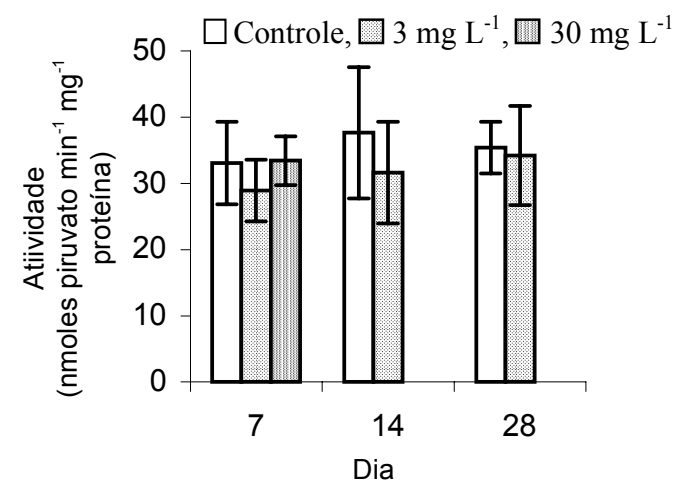

Figura 1 - Atividade de TGO em músculo de peixes expostos ao paclobutrazol. 


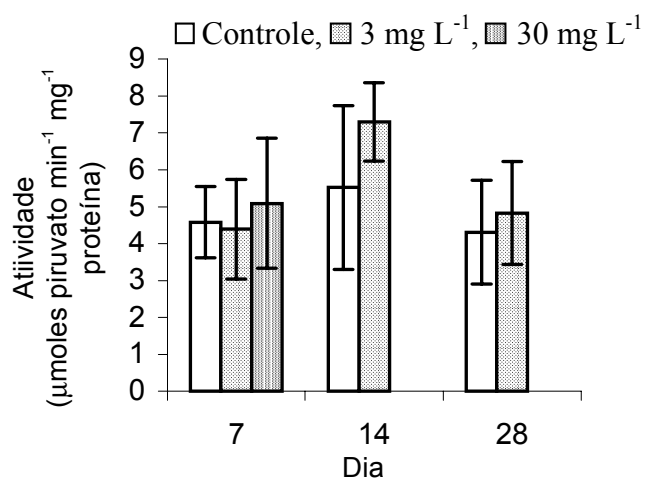

Figura 2 - Atividade de TGO em fígado de peixes expostos ao paclobutrazol.

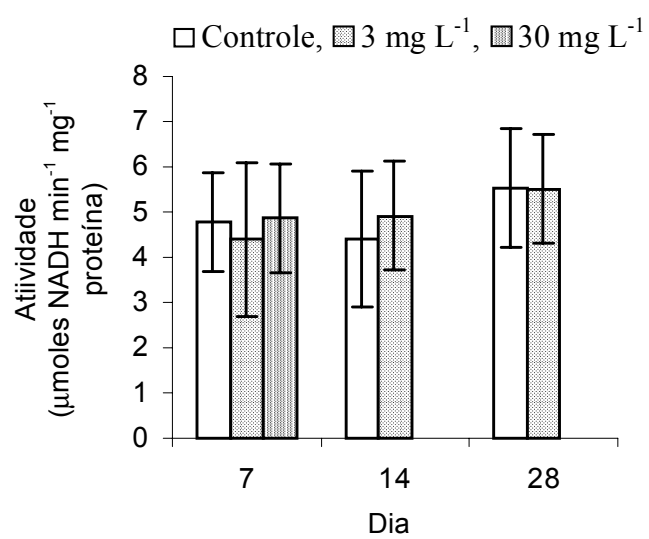

Figura 3 - Atividade de LDH em músculo de peixes expostos ao paclobutrazol.

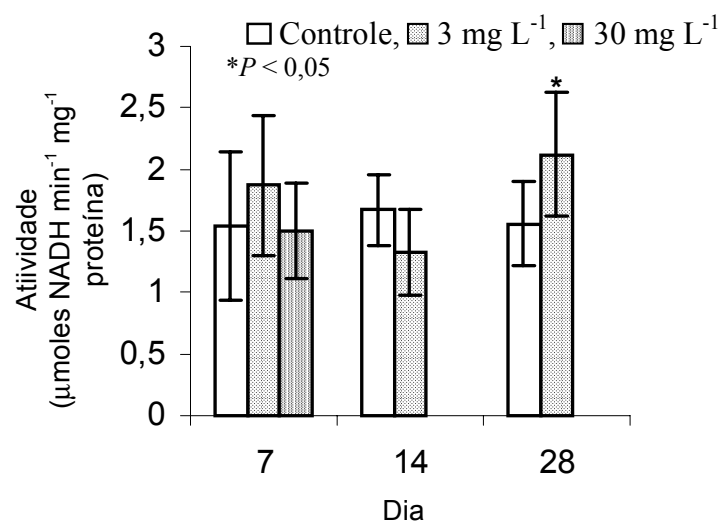

Figura 4 - Atividade de LDH em fígado de peixes expostos ao paclobutrazol.

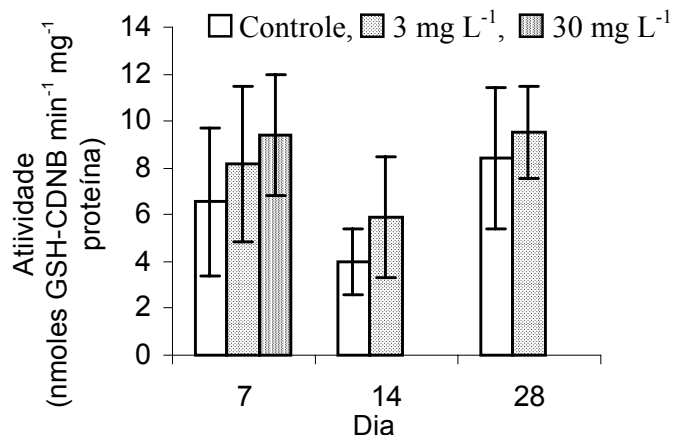

Figura 5 - Atividade de GST em músculo de peixes expostos ao paclobutrazol.

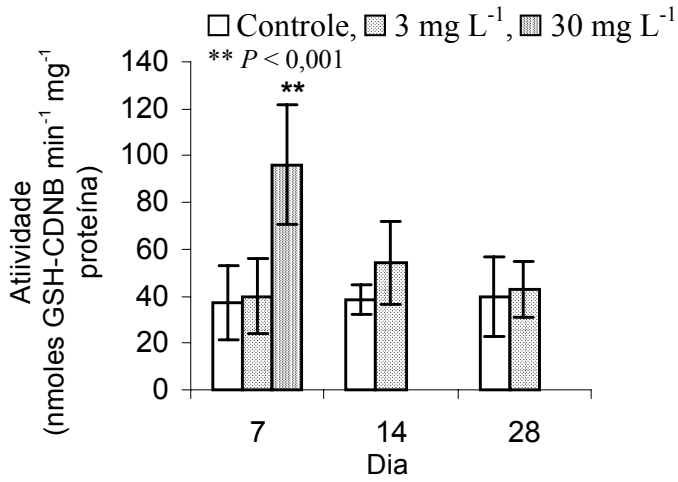

Figura 6 - Atividade de GST em fígado de peixes expostos ao paclobutrazol.

Embora as transaminases sejam induzidas por vários compostos em condições de exposição crônica, isto não se manifesta para alguns agentes, incluindo agroquímicos, tal como demostraram os nossos resultados. Neskovic et al. (1993) não observaram alterações nas atividades de transaminases em fígado de carpas (Cyprinus carpio) expostas a 3,0 e $6,0 \mathrm{mg} \mathrm{L}^{-1}$ de atrazina (correspondente a 1/6 e 1/3 LC50-96h, respectivamente) por 14 dias. Durante uma exposição crônica ao cádmio na concentração de $12,7 \mathrm{mg} \mathrm{L}^{-1}$, embora se tenha observado a mortalidade do peixe Lepomis macrochirus, não foram observadas alterações nas atividades de TGO e transaminase glutâmico pirúvica (TGP) durante o período de 32 dias (Versteeg \& Giesy, 1986).

A literatura relata que assim como o paclobutrazol, outros compostos com propriedades fungicida foram incapazes de alterar a TGO em peixes sob condições experimentais. Como exemplo, o fungicida pentaclorofenol, na concentração de de 0,1 e 0,2 mg $\mathrm{L}^{-1}$, não alterou os níveis séricos de TGO em Leuciscus idus melanotus L. após quatro horas, mas a concentração sérica da enzima foi alterada por outros fungicidas fenólicos (Tiedge et al., 1986) e inorgânicos (Rojik et al., 1983).

Samuel \& Sastry (1989) não observaram efeitos na atividade de $\mathrm{LDH}$ em fígado e músculo do peixe Channa punctatus exposto a $1 \mathrm{mg} \mathrm{L}^{-1}$ (1/10CL50-96h) do inseticida monocrotofós durante duas semanas, sendo que o aumento da atividade se evidenciou aos 30 dias de exposição. O aumento de LDH após o $28^{\circ}$ dia de exposição indica que o peixe se utilizou de mecanismos anaeróbicos de respiração de modo a suprir a demanda de energia quando a oxidação aeróbica está comprometida. Apesar deste mecanismo de compensação também ocorrer no tecido muscular, os nossos resultados não evidenciaram alterações na atividade de LDH neste tecido (Figura 3).

A enzima GST desempenha um papel importante na detoxificação e eliminação de compostos eletrofílicos, incluindo agroquímicos. Sua estimulação envolve reações de conjugação na presença de glutationa. Assim, animais aquáticos que habitam ambientes 
poluidos podem estar expostos a xenobióticos os quais sofrem detoxificação mediada pela glutationa na sua forma reduzida, catalisada pela enzima glutationa Stransferase. Esta enzima de biotransformação tem sido estudada em trabalhos de campo no monitoramento de poluentes de origem industrial (Fenet et al. 1998; Kantoniemi et al., 1996) e agrícola (Cho et al., 1999).

Outros autores também relataram a detoxificação de outros compostos com propriedades fungicidas realizada pela GST. Gallagher \& Di Giulio (1992) observaram que a metabolização do clorotalonil de frações do citosol de brânquias do peixe Ictalurus punctatus foi diminuída na ausência de glutationa reduzida. Egaas et al. (1999) mostraram que o propiconazole induz a atividade da GST hepática em trutas (Salmo trutta) na concentração de 8,3 - $606 \mu \mathrm{g} \mathrm{L}^{-1}$, e que esta indução é dependente da dose e peso corporal do organismo teste.

Em vista dos resultados do presente trabalho, a fase II do metabolismo, envolvendo reações de conjugação, deve ser um dos processos na conversão do paclobutrazol em compostos mais facilmente eliminados. À semelhança do observado para a LDH, a indução de GST foi manifestada no tecido hepático, não se constatando um aumento de sua atividade no tecido muscular (Figura 5). Esta suposição é aceitável já que o fígado é o principal órgão de biotransformação de xenobióticos em seres vivos. Otto \& Moon (1995) obtiveram respostas semelhantes aos nossos resultados após o tratamento de peixes com 3,3',4,4'-tetraclorobifenil, o qual induziu a atividade de GST hepática em aproximadamente duas vezes, o mesmo não ocorrendo no tecido muscular.

As alterações ocorridas na atividade da GST e LDH sugerem que a medida da atividade destas enzimas na espécie testada poderia ser usada como um biomarcador associado à contaminação aquática por paclobutrazol, mas haveriam algumas limitações quanto à implementação de seu uso. Primeiramente, a atividade de GST aumentou em concentrações relativamente altas do composto na água, as quais seriam atingidas somente quando se ultrapassasse em muitas vezes a dose de aplicação agronômica, o que poderia limitar a aplicabilidade de seu uso em programas de monitoramento ou avaliação de risco. Por outro lado, embora tenha se constatado um aumento da atividade de LDH para a menor concentração testada, esta indução se manifestou somente num período superior a duas semanas de exposição, o que limitaria a obtenção de resultados a curto prazo. A ausência de alterações na atividade da TGO sugere que a medida da mesma não seria útil como indicadora da poluição aquática pelo paclobutrazol e que este composto não estaria interferindo numa das importantes fases de transaminação no metabolismo de aminoácidos.

$\mathrm{Na}$ Figura 7 estão apresentados os resultados referentes ao estudo da bioconcentração de paclobutrazol em Metynnis argenteus. Os valores correspondem à média de 2 análises + desvio padrão.
Para 7 e 14 dias os valores para o músculo correspondem a uma determinação. No final da exposição, a concentração de paclobutrazol determinada em músculo atingiu níveis residuais de $166 \mathrm{mg} \mathrm{kg}^{-1}$ para a concentração média de $2,7 \mathrm{mg} \mathrm{L}^{-1}$ determinada na água $(F B C=61)$. O FBC estimado para o paclobutrazol na situação de equilíbrio foi equivalente a 73 . Este valor é muito próximo ao calculado a partir dos resultados de resíduos de paclobutrazol em músculo do peixe Carassius auratus relatados por Liu et al. (1996).

Comparando-se o valor de FBC calculado para paclobutrazol com os de outros compostos com propriedades fungicidas, observa-se que o mesmo apresenta ordem de grandeza semelhante àquelas relatadas na literatura. Assim, Tsuda et al. (1992) relataram um FBC equivalente a 25 e 100, respectivamente, para o clorotalonil e captan no peixe Gnathopogon caerulescens. $\mathrm{O}$ tiabendazol demonstrou ter um FBC equivalente a 23 no tecido muscular de Lepomis macrochirus (Van den Heuvel et al., 1997). Os valores de FBC calculados para isoprothiolane, iprobenfós, edifenfós e carbendazim em diversas espécies de peixes foi equivalente a 45 (Tsuda et al.,1997), 26 (Tsuda et al., 1990), 110 (Tsuda et al., 1994) e 27 (WHO Working Group, 1993), respectivamente. Porém, o paclobutrazol apresentou valor de FBC de 2 a 6 vezes menor que o estimado para o benomil em outras espécies de peixes (U.S. National Library of Medicine, 1995; Howard, 1991).

O baixo potencial de acúmulo do paclobutrazol em tecidos, quando comparado a valores de FBC muito mais elevados de outros fungicidas, tal como é o caso do pentaclorofenol (FBC = 1680; Tachikawa et al, 1991), parece não estar associado aos valores de coeficiente de partição octanol/água $\left(\mathrm{K}_{\mathrm{ow}}\right)$, visto que são descritos valores deste parâmetro físico-químico equivalentes a 1590 (Worthing \& Hance, 1991) e 5,12 (U.S. National Library of Medicine, 1995), respectivamente, para o paclobutrazol e para o pentaclorofenol.

De acordo com valor estimado de FBC, e com o valor da Ingestão Diária Aceitável (IDA) para o paclobutrazol de $0,1 \mathrm{mg} \mathrm{kg}^{-1}$ de peso corpóreo (Worthing \& Hance, 1991), a concentração máxima permitida do

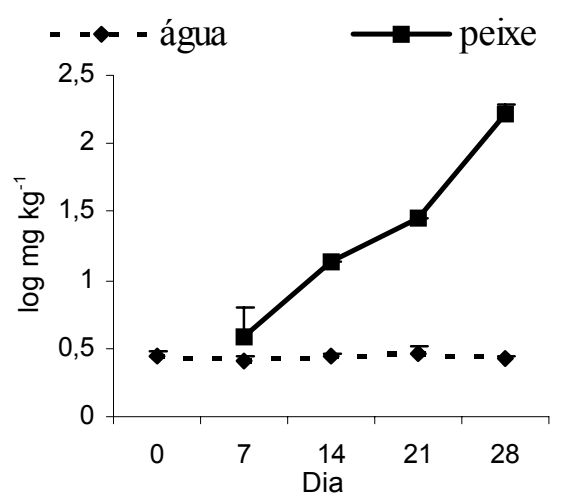

Figura 7 - Concentração de paclobutrazol em músculo e água em função do tempo. 
ingrediente ativo em corpos de água para evitar efeitos adversos quanto ao consumo do peixe seria equivalente a $0,2 \mathrm{mg} \mathrm{L}^{-1}$. Este valor hipotético foi estimado considerando-se o peso de um indivíduo adulto de 70 $\mathrm{kg}$ que consumiria diariamente $500 \mathrm{~g}$ do peixe, sendo que essa concentração na água seria atingida pela aplicação direta da dose máxima recomendada do paclobutrazol $\left(3,0 \mathrm{~kg}\right.$ i.a. ha ${ }^{-1)}$ sobre uma lâmina de água de $150 \mathrm{~cm}$.

\section{CONCLUSÕES}

A exposição dos organismos-teste ao paclobutrazol alterou a atividade de LDH e GST, sugerindo a medida da atividade destas enzimas como biomarcadoras de exposição ao composto; a biotransformação do paclobutrazol em compostos mais facilmente eliminados envolve reações de conjugação com glutationa e que o paclobutrazol se acumula no tecido muscular do peixe em função do tempo de exposição, mas em função do baixo fator de bioconcentração do composto neste tecido, haveria baixo risco para a saúde humana quanto ao consumo de pescado, a não ser em situações extremas de exposição dos organismos aquáticos ao produto.

\section{REFERÊNCIAS BIBLIOGRÁFICAS}

ASZTALOS, B.; NEMCSOK, J.; BENEDECZKY, R.G.; SZABO, A.; REFAIE, O.J. The effects of pesticides on some biochemical parameters of carp (Cyprinus carpio L.) Archives of Environmental Contamination and Toxicology, v.19, p.275-282, 1990.

BUCHELI, T.D.; FENT, K. Induction of citochorome P450 as a biomarker for environmental contamination in aquatic ecosystems. Critical Reviews in Environmental Science and Technology, v.25, p.201268, 1995.

CHAND, T.; LEMBI, C.A. Dissipation of gibberelin synthesis inhibitors in small-scale aquatic systems. Journal of Aquatic Plant Managenment, v.22, p.15-20, 1994.

CHO J.R.; KIM Y. J.; HONG K. J.; YOO J. K.; LEE J.O.; AHN Y. J.; CHO J.R.; KIM Y.J.; HONG K.J.; YOO J.K.; LEE J.O.; AHN Y.J. Resistance monitoring and enzyme activity in field-collected populations of the spiraea aphid, Aphis citricola Van der Goot. Journal of Asian Pacific Entomology, v. 2, p.113-119, 1999.

EGAAS, E.; SANDVICK, M.; FJELD, E.; KALLQVIST, T.; GOKSOYR, A.; SVENSEN, A. Some effects of the fungicide propiconazole on cytochrome P450 and glutathione S-transferase in brown trout (Salmo trutta). Comparative Biochemistry and Physiology C; Pharmacology, Toxicology and Endocrinology, v.122, p.337-344, 1999.

FENET, H.; CASELLAS, C.; BONTOUX, J. Laboratory and field caging studies on hepatic enzymatic activities in european eel and rainbow trout. Ecotoxicology and Environmental Safety, v.40, p.137-143,1998.

FRANKEL, S.; REITMAN, S. Granwhol's clinical laboratory and diagnosis. 6.ed. London: The C.V. Mosby, 1963. v.1, 222p.

GALLAGHER, E.P.; DI GIULIO, R.T. Glutathione mediated chlorothalonil detoxification in channel catfish gills. Marine Environmental Research, v.34, p.221-226, 1992.

HABIG, W.H.; PABST, M.J.; JAKOBY, W.B. Glutathione S-transferases. The first enzimatic step in mercapturic acid formation. The Journal of Biogical Chemistry, v.249, p.7130-7139, 1974.

HOWARD, P. H. Handbook of environmental fate and exposure data for organic chemicals: Pesticides. Chelsea: Lewis Publishers, 1991. v.3.

JIMENEZ, B.D.; STEGEMAN, J.J. Detoxication enzymes as indicators of environmental stress on fish. American Fisheries Society Symposium, v.8, p.67-69, 1990.

KANAZAWA, J. Measurement of the bioconcentration factors of pesticides by freshwater fish and their correlation with phisicochemical properties or acute toxicities. Pesticide Science, v.12, p.417-424, 1981.
KANTONIEMI, A.; KIRSI, V.; OIKARI, A. The capacity of liver microsomes to form benzo(a)pyrene-diolepoxide -DNA adducts and induction of cytochrome P450 1A in feral fish exposed to pulp mill effluents. Ecotoxicology and Environmental Safety, v.35, p.136-141, 1996.

LIU, Z.T.; KONG, M.Z.; ZHOU, F.; WANG, S.L. Bioconcentration and toxicity effect on lipid content of aquatic organisms. Bulletin of Environmental Contamination and Toxicology, v.56, p.135-142, 1996.

MEHRLE, P.M.; MAYER, F.L. Biochemistry/Physiology. In: RAND, G.R.; PETROCELLI, S.R. Fundamentals of aquatic toxicology. New York: Hemisphere Publishing Corporation, 1985. p.264-282.

NESKOVIC, N.K. ; ELEZOVIC, I.; KARAN, V.; POLEKSIC, V.; BUDIMIR, M. Acute and subacute toxicity of atrazine to carp. Ecotoxicology and Environmental Safety, v.25, p.173-182, 1993.

OTTO, D.M.E.; MOON, T.W. 3,3',4',4'-tetrachlorobiphenyl effects on antioxidants enzymes and glutathione status in different tissues of rainbow trout. Pharmacology and Toxicology, v.77, p.281-287, 1995.

PETERSON, G.L. A simplification method of the protein method of Lowry et al. which is more generally applicable. Analytical Biochemistry, v.83, p.346-356, 1977

RAO, R.V.K.; SURENDRANATH, P.; KODAVANTI, P.R.S. Levels of transaminases in tissues of the penaeid prawn, Metapenaeus monoceros (Fabricius) following sublethal kelthane exposure. Bulletin of Environmental Contamination and Toxicology, v.44, p.114-120, 1990.

REDDY, A.N.; VENUGOPAL, N.B.R.K.; REDDY, S.L.N. Effect of endosulfan $35 \mathrm{EC}$ on some biochemical changes in the tissues and haemolymph of a freshwater field crab, Barytelphusa guerini. Bulletin of Environmental Contamination of Toxicology, v.55, p.116-121, 1995.

ROJIK, I.; NEMSOK, J.; BOROSS, L. Morphological and biochemical studies on liver, kidney and gill of fishes affected by pesticides. Acta Biologica Hungarica. v.34, p.81-92, 1983.

SAMUEL, M.; SASTRY, K.V. In vivo effect of monocrotophos on the carbohydrate metabolism of the freshwater snake head fish; Channa punctatus. Pesticide Biochemistry and Physiology, v.34, p.1-8, 1989.

SPACIE, A.; HAMELINK, J.L. Bioaccumulation. In: RAND, G.R.; PETROCELLI, S.R. Fundamentals of aquatic toxicology. New York: Hemisphere Publishing Corporation, 1985. p.495-525.

TACHIKAWA, M.; SAWAMURA, R.; OKADA, S.; HAMADA, A. Differences between freshwater and seawater killifish (Oryzias latipes) in the accumulation and elimination of pentachlorophenol. Archives of Environmental Contamination and Toxicology, v.21, p.146-151, 1991.

TIEDGE, H.; NAGEL, R.; URICH, K. Effect of substituted phenols on transaminase activity in the fish, Leuciscus idus melanotus L. Bulletin of Environmental Contamination and Toxicology, v.36, p.176-180, 1986.

TSUDA, T.; AOKI, S.; KOJIMA, M.; FUJITA, T. Accumulation and excretion of pesticides used in golf courses by carp (Cyprinus carpio) and willow shiner (Gnathopogon caerulescens). Comp. Biochem. Physiol. C Comp. Pharmacology and Toxicology, v. 101, p. 63-66, 1992.

TSUDA, T.; AOKI, S.; KOJIMA, M.; FUJOTA, T. Pesticides in water and fish from rivers flowing into Lake Biwa (III). Toxicological and Environmental Chemistry, v.41, p.85-90, 1994.

TSUDA, T.; AOKI, S.; KOJIMA, M.; HARADA, H. Bioconcentration and excretion of diazinon, IBP, malathion and fenitrothion by carp. Comparative Biochemistry and Physiology C, Comparative Pharmacology and Toxicology, v.96, p.23-26, 1990.

TSUDA, T.; KOJIMA, M.; HARADA, H.; NAKAJIMA, A.; AOKI, S. Acute toxicity, accumulation and excretion of isoprothiolane and its degradation products in killifish. Water Research, v.31, p.323-327, 1997.

U.S. NATIONAL - LIBRARY OF MEDICINE. Hazardous substances data bank. Bethesda, 1995.

VANDEN HEUVEL, W.J.A.; WISLOCKI, P.G.; HIRSCH, M.P.; PORTER, N.K.; AMBROSE, R.T.; ROBILLARD, K.A. Bioconcentration and metabolism of thiabendazole [2-(4-thiazolyl)-1H-benzimidazole] in bluegill sunfish, Lepomis macrochirus. Journal of Agricultural and Food Chemistry, v.45, p.985-989, 1997.

VASSAULT, A. Lactate dehidrogenase. In: BERGMEYER, H.V. (Ed.) Methods in enzimatic analysis. Weinheim: Verlag Chemie, 1983. v.3, p.118-126.

VERSTEEG, D.J.; GIESY, J.P. The histological and biochemical effects of cadmium exposure in bluegill sunfish (Lepomis macrochirus). Ecotoxicology and Environmental Safety, v.11, p.31-43, 1986.

WHO WORKING GROUP. Benomyl. Geneva: WHO, 1993. 135p. (Environmental Health Criteria, 148)

WORTHING, C.R.; HANCE, R.J. The pesticide manual. 9.ed. Surrey: The British Crop Protection Council, 1991. 644p.

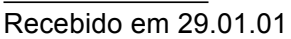

